\title{
THE HOMEOMORPHISM GROUP OF A COMPACT $Q$-MANIFOLD IS AN ANR
}

BY STEVE FERRY

\author{
Communicated by T. A. Chapman, June 1, 1976
}

In this paper we will let $Q=\Pi_{i=1}^{\infty}[-1,1]$ denote the Hilbert cube, $l_{2}=$ $\left\{\left.\left(x_{i}\right)|\Sigma| x_{i}\right|^{2}<\infty\right\}$ denote separable Hilbert space, $C(X, X)$ denote the continuous functions from $X$ to $X$ with the sup norm topology with respect to a suitable metric on $X, H(X) \subset C(X, X)$ denote the self-homeomorphisms of $X$, and $\bar{H}(X)$ denote the closure of $H(X)$ in $C(X, X)$. By a manifold, we will mean a separable metric space modelled on $\mathbf{R}^{n}, Q$, or $l_{2}$. The following question is of interest to both infinite dimensional and finite dimensional topologists. Compare $[\mathbf{A}-\mathbf{B}, \mathrm{p}$. 792].

Q1. If $M$ is a manifold modelled on $\mathbf{R}^{n}$ or $Q$, is $H(M)$ an $l_{2}$-manifold?

General results which are known include:

(1) Geoghegan [G] has shown that $H_{A}(M) \times l_{2} \stackrel{\text { hoom }}{\approx}{ }^{\circ} H_{A}(M)$. Here, $H_{A}(M)$ is the space of homeomorphisms of $M$ which fix a proper closed subset $A$ of $M$.

(2) Toruncyzk [T] has shown that the product of $l_{2}$ with a complete separable metric ANR is an $l_{2}$-manifold.

These results reduce Q1 to the question:

Q1'. If $M$ is a compact manifold, is $H(M)$ an ANR?

(3) Mason and Luke and Mason [M] , [L-M] have answered Q1' affirmatively when $M$ is a two-dimensional manifold.

Using completely different techniques, we have proven the analogous result for $Q$-manifolds. Recall that a closed set $A \subset M$ is called a $Z$-set if there are maps of $M$ into $M-A$ which are arbitrarily close to the identity.

THEOREM 1. If $M$ is a compact $Q$-manifold and $A \subset M$ is a Z-set, then $H_{A}(M)$ is an $A N R$.

We require more notation in order to state our next result. Let $X$ and $Y$ be metric spaces, and let $\beta$ be an open cover of $Y$. A homotopy $H: X \times I \rightarrow Y$ is called a $\beta$-homotopy if for each $x \in X$ there is an element $U_{x} \in \beta$ such that

AMS (MOS) subject classifications (1970). Primary 54C55, 57A20, 57C10, 58D05; Secondary 57A60, 57E05.

Key words and phrases. Simple homotopy, $Q$-manifold, $l_{2}$-manifold, $C E$ approximation, homeomorphism group. 
$H(\{x\} \times I) \subset U_{x}$. A map $f: X \rightarrow Y$ will be called a $\beta$-equivalence if there is a map $g: Y \rightarrow X$ such that $f \circ g$ is $\beta$-homotopic to the identity and $g \circ f$ is $f^{-1}(\beta)$ homotopic to the identity. $f$ is called a fine homotopy equivalence if $f$ is a $\beta$ equivalence for each open cover $\beta$ of $Y$.

Basic work on fine homotopy equivalences has been done by Lacher, Price, Kozlowski, and Haver, among others.

If $f, g: X \rightarrow Y$ are maps and $\beta$ is an open cover of $Y, f$ and $g$ are said to be $\beta$-close if for each $x \in X$ there is an open $U_{x} \in \beta$ such that $f(x), g(x) \in U_{x}$. A map $f: X \rightarrow Y$ is called a near-homeomorphism if for each open cover $\beta$ of $Y$ there is a homeomorphism $h: X \rightarrow Y$ which is $\beta$-close to $f$. If $M$ is compact, then $f: M \rightarrow M$ is in $\overline{H(M)}$ if and only if $f$ is a near-homeomorphism.

Armentrout [A], Siebenmann [S], and Chapman $\left[\mathrm{Ch}_{\mathbf{1}}\right]$ have proven (roughly) that the near-homeomorphisms are the fine homotopy equivalences between $n$-manifolds of dimensions $n \leqslant 3, n \geqslant 5$, and for $Q$-manifolds, respectively. For exact statements of the theorems, consult the references cited above.

Our main tool in the proof of Theorem 1 is a parametrized estimated version of Chapman's theorem. We state the corresponding unparametrized result as Theorem 2. Recall that a map $f: X \rightarrow Y$ is proper if $f^{-1}(K)$ is compact for each compact $K \subset Y$. A $\beta$-equivalence $f$ is proper if $f, g$ and the homotopies are proper.

THEOREM 2. If $M$ is a $Q$-manifold and $\alpha$ is an open cover of $M$, then there is an open cover $\beta$ of $M$ such that if $N$ is a $Q$-manifold and $f: N \rightarrow M$ is a proper $\beta$-equivalence then $f$ is $\alpha$-close to a homeomorphism.

Note that $\beta$ depends on $M$ and $\alpha$ but not on $N$. As an immediate corollary, we obtain

THEOREM $2^{\prime}$. If $K$ is a countable locally finite polyhedron, then there is an open cover $\beta$ of $K$ such that if $f: L \rightarrow K$ is a proper $\beta$-equivalence then $f$ is a simple homotopy equivalence.

This is a considerable generalization of the theorem that $C E$ maps between locally finite polyhedra are simple homotopy equivalences $\left[\mathrm{Ch}_{\mathbf{2}}\right]$.

The techniques of our proof yield an even stronger result for $l_{2}$-manifolds.

THEOREM 3. If $M$ and $N$ are $l_{2}$-manifolds, $\alpha$ is an open cover of $M$, and $f: N \rightarrow M$ is an $\alpha$-equivalence, then $f$ is St $\alpha$-close to a homeomorphism.

Thus, in the category of $l_{2}$-manifolds, the near-homeomorphisms are precisely the fine homotopy equivalences.

\section{REFERENCES}

[A-B] R. D. Anderson and R. H. Bing, A complete elementary proof that Hilbert space is homeomorphic to the countable infinite product of lines, Bull. Amer. Math. Soc. 74 (1968), 771-792. MR 37 \#5847. 
[A] S. Armentrout, Concerning cellular decompositions of 3-manifolds that yield 3manifolds, Trans. Amer. Math. Soc. 133 (1968), 307-332. MR 37 \#5859.

[Ch $\mathrm{Ch}_{1}$ T. A. Chapman, Cell-like mappings of Hilbert cube manifolds: Solution of a handle problem, General Topology and Appl. 5 (1975), 123-145.

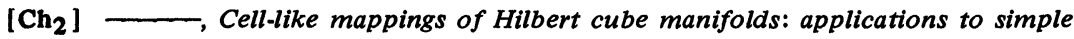
homotopy theory, Bull. Amer. Math. Soc. 79 (1973), 1286-1291. MR 48 \#5083.

[G] R. Geoghegan, On spaces of homeomorphisms, embeddings and functions. I, Topology 11 (1972), 159-177. MR 45 \#4349.

[L-M] R. Luke and W. K. Mason, The space of homeomorphisms on a compact twomanifold is an absolute neighborhood retract, Trans. Amer. Math. Soc. 164 (1972), 275285. MR $46 \# 849$.

[M] W. K. Mason, The space of all self-homeomorphisms of a two-cell which fix the cell's boundary is an absolute retract, Trans. Amer. Math. Soc. 161 (1971), 185-205. MR 44 \#3283.

[S] L. C. Siebenmann, Approximating cellular maps by homeomorphisms, Topology 11 (1972), 271-294. MR 45 \#4431.

[T] H. Toruńczyk, Absolute retracts as factors of normed linear spaces, Fund. Math. 86 (1974), 53-67. MR 51 \#1723.

DEPARTMENT OF MATHEMATICS, UNIVERSITY OF KENTUCKY, LEXINGTON, KENTUCKY 40506 